\title{
Special Section Guest Editorial: Representation Learning and Big Data Analytics for Remote Sensing
}

\author{
Weifeng Liu, ${ }^{\text {a }}$ Yicong Zhou, ${ }^{\text {b }}$ Karen Panetta, ${ }^{\mathrm{c}}$ and Sos Agaian ${ }^{\mathrm{d}}$ \\ ${ }^{a}$ China University of Petroleum (East China), College of Control Science and Engineering, \\ Qingdao, China \\ ${ }^{b}$ University of Macau, Department of Computer and Information Science, Macao, China \\ ${ }^{\mathrm{c}}$ Tufts University, Department of Electrical and Computer Engineering, Medford, \\ Massachusetts, United States \\ ${ }^{\mathrm{d} C}$ City University of New York, College of Staten Island, New York, United States
}

With the rapid growth of remote sensing data delivered by remote sensors, it becomes increasingly critical to explore the efficient and effective artificial intelligence methodologies and data science techniques for complex information retrieval in remote sensing. Although many promising achievements were reported for signal and image processing applications, it is still a great challenge to develop distinctive algorithms for unique remote sensing analytics.

Recently, representation or feature learning draws a widely public attention and plays an important role for signal and image analysis. There are some successful examples of representation learning, e.g. graph convolutional networks have dramatically improved the performance of image classification; self-representation learning methods have been applied for segmentation and clustering; spatial convolution related methods have been successfully applied to semantic segmentation and region recognition; long short term memory networks are successfully used in relation extraction and multi-hop reading comprehension.

This special section aims to demonstrate the contribution of representation learning algorithms to the research of remote sensing analytics. It contains 11 independent studies in the related aspects of representation learning for remote sensing: (1) feature extraction, (2) object/image recognition/classification, (3) image super-resolution, and (4) novel experimental design and algorithm development. Min Xia et al. employed residual neural network to obtain multi-scale features for river segmentation. Xiaofei Mi et al. proposed a multiscale stacked denoising autoencoder model to extract urban build-up area features in different scales and recognize the type of land object from multiple scales. Min Xia et al. constructed a multiscale fusion attention network to recognize cloud and snow areas in plateau remote sensing images. Daizhi Kuang and Juncai Xu combined multiple spectral-spatial features and multikernel support tensor machine for hyperspectral image classification. Yanshan Li et al. built a tensor-based spatialspectral local binary pattern to utilize the local spatial-spectral feature of hyperspectral images and improved the classification accuracy significantly. Ernest D. Ganaa et al. presented a robust deflated principal component analysis via multiple instance factorings for dimension reduction in remote sensing images. Sarwar Shah Khan et al. proposed an NRS-Manhattan distance strategy into nearest regularized subspace for hyperspectral image classification. Beatriz P. P. GarciaSalgado et al. provided some analysis of dimension reduction of hyperspectral images for big data remote sensing applications. Poppy Immel et al. presented deep learning model selection for angular feature extraction in satellite imagery. Deepakrishna Somasundaram et al. implemented vector quantization neural network-based method for surface water extraction from Landsat OLI images. Junwei Zhang et al. presented a satellite image super-resolution based on progressive residual deep neural network.

These studies indicate that representation learning (e.g. feature extraction) plays an essential role for segmentation, recognition, and classification of remote sensing images. And big data analytics is becoming more popular in remote sensing applications. Although a single-view feature (e.g. dimension reduction, local binary patterns) is reported in some studies, a multiview feature (e.g. multiscale or spectral-spatial feature) improves performance significantly and

(C) 2020 Society of Photo-Optical Instrumentation Engineers (SPIE) 
becomes more attractive. It is critical to develop comprehensive theories and algorithms for representation learning and big data analytics in remote sensing.

Finally, we would like to thank the authors of the special section for their contributions. Great appreciations to the reviewers and the Journal of Applied Remote Sensing editorial team for conducting a high-quality review process for all of the published papers. We hope that this special section will foster further development of representation learning and big data analytics in remote sensing. 